\title{
Marjorie Curry woods, Weeping for Dido: The Classics in the Medieval Classroom
}

\section{Anne-Lydie Dubois}

\section{(2) OpenEdition}

\section{Journals}

Édition électronique

URL : https://journals.openedition.org/ccm/7008

DOI : $10.4000 / \mathrm{ccm} .7008$

ISSN : 2119-1026

\section{Éditeur}

Centre d'études supérieures de civilisation médiévale/Université de Poitiers

\section{Édition imprimée}

Date de publication : 1 janvier 2021

Pagination : 99-101

ISBN : 978-2-490783-08-3

ISSN : 0007-9731

Référence électronique

Anne-Lydie Dubois, "Marjorie Curry woods, Weeping for Dido: The Classics in the Medieval Classroom», Cahiers de civilisation médiévale [En ligne], 253 | 2021, mis en ligne le 01 mars 2021, consulté le 23 novembre 2022. URL : http://journals.openedition.org/ccm/7008; DOI : https://doi.org/10.4000/ccm. 7008

\section{(c) (i) (3)}

Creative Commons - Attribution - Pas d'Utilisation Commerciale - Pas de Modification 4.0 International - CC BY-NC-ND 4.0

https://creativecommons.org/licenses/by-nc-nd/4.0/ 
Marjorie Curry Woods, Weeping for Dido: The Classics in the Medieval Classroom, Princeton, Princeton University Press (E. H. Gombrich Lecture Series), 2019.

L'étude que propose Marjorie Curry Woods se penche sur les pratiques d'apprentissage des écoliers du Moyen Âge, en portant son attention sur les émotions que véhiculent les textes classiques utilisés dans ce contexte à travers les commentaires laissés dans les manuscrits. Elle suggère que la charge émotionnelle que contiennent les œuvres apprises dans les salles de classe médiévales constitue un formidable outil pédagogique pour ancrer les leçons dans la mémoire des écoliers. Ces textes étant récités à haute voix, voire joués dans les classes, proposent une distorsion de genre, une forme de transgression, à travers leur lecture. En effet, les écoliers, exclusivement masculins, qui composent les classes intègrent et transmettent les sentiments des personnages féminins qu'ils incarnent par ce biais, comme ceux de la figure emblématique de la reine Didon. À travers le pathos auquel ils donnent vie en jouant les textes classiques, les étudiants donnent corps aux émotions du sexe opposé comme le souligne M. C. Woods. Dans Weeping for Dido: The Classics in the Medieval Classroom, la perspective du genre est associée à l'étude des sentiments afin de faire de la réception des textes classiques un biais pour l'étude de la littérature médiévale ainsi que pour l'histoire culturelle de cette période. La classe se fait ainsi communauté émotionnelle à l'intérieur de laquelle se vivent des sentiments fictionnels qui permettent aux jeunes élèves de s'identifier aux personnages par cette expérience sociale partagée.

L'approche mise en œuvre dans Weeping for Dido, à travers les questions soulevées et les analyses proposées, est résolument interdisciplinaire. La recherche de M. Curry Woods se situe à la croisée de l'étude de la littérature et de l'histoire de l'éducation, qu'elle associe par la perspective du genre et des émotions. En plein essor, ces deux derniers domaines ont donné lieu à de fructueux travaux ces dernières années. Si l'utilité de ces approches n'est plus à démontrer, l'originalité du travail de l'a. réside dans la conjonction de ces différents axes de recherche, employant l'étude des manuscrits et des gloses qu'ils contiennent, du point de vue de leur matérialité et de leur contenu, afin de saisir la réception des textes classiques au Moyen Âge. M. C. Woods a consacré de nombreux travaux aux techniques pédagogiques médiévales, en relation avec l'apprentissage de l'art poétique et de la rhétorique. Son ouvrage paru en 2010, Classroom Commentaries: Teaching the Poetria nova across Medieval and Renaissance Europe (Colombus, Ohio State University Press [Text and Context]), se penchait déjà sur ces-questions. Weeping for Dido s'inscrit dans ce prolongement, en ajoutant la 
dimension du genre et celle des émotions en tant que biais pour aborder cette thématique. Il s'agit de prendre en considération ce que l'on pourrait qualifier d'émotions «genrées », ou plutôt d'émotions attribuées à des personnages fictifs féminins, afin de comprendre leur rôle primordial dans l'intégration de la matière transmise. Le prisme du genre ne se limite toutefois pas au féminin car l'étude prend également en considération la masculinité. Ce domaine de recherche au développement récent pour les études médiévales, en regard de l'histoire des femmes, est ici pleinement intégré à l'approche de la littérature que propose M. C. Woods. Les émotions féminines et masculines, ainsi que la féminité et la masculinité en tant qu'identités de genre, sont notamment considérées à travers la question de l'inversion des sexes que suggèrent les différents moyens d'identification mis en œuvre par les textes classiques et leurs commentaires. Ce phénomène se produit lorsque les écoliers incarnent les personnages féminins à la lecture des textes, mais aussi par la mise en exergue du travestissement d'Achille, suggérant une identification de genre troublée.

Weeping for Dido, dont le titre souligne toute l'empathie émotionnelle qu'inspirent les textes classiques, propose une analyse minutieuse à partir de plusieurs œuvres littéraires transmises par les manuscrits latins des derniers siècles du Moyen Âge. Un travail de recherche conséquent et rigoureux est mis au jour par l'étude d'un grand nombre de manuscrits, provenant d'Allemagne, d'Italie, d'Angleterre ou encore de France, ainsi que de leurs gloses interlinéaires et marginales. Soixante manuscrits de l'Énéide, cinquante de l'Achilléide et trente de l'Iliade latine (Ilias latina), soit l'Homère latin, répartis sur un arc chronologique s'échelonnant entre le XII et le $\mathrm{XV}^{\mathrm{e}} \mathrm{s}$., ont ainsi été scrutés par M. C. Woods. L'ouvrage se divise en trois chapitres qui proposent chacun une facette différente du processus pédagogique et du rapport d'identification des lecteurs mis en lumière par les manuscrits des textes classiques. Trois textes structurent les deux premiers chapitres. La réception de l'Énéide de Virgile au Moyen Âge fait l'objet du premier, qui se penche sur les émotions de Didon soulignées par les gloses, tandis que le second s'articule autour de l'Achilléide et de l'Iliade latine, afin de se concentrer sur la question de la masculinité. Le troisième et dernier chapitre s'intéresse quant à lui à la question de la performance des textes récités par le biais des instructions aux lecteurs présentes dans les manuscrits médiévaux.

L'expérience de saint Augustin en tant qu'écolier à l'âge de douze ans sert de point de départ à l'étude de l'identification des jeunes hommes aux émotions féminines véhiculées par les textes classiques. Instruit dans sa jeunesse à travers les textes païens et l'œuvre de Virgile en particulier, il raconte dans les Confessions comment il pleura avec Didon. Pour M. C. Woods, les fortes émotions ressenties à l'égard de la reine à la lecture de l'Énéide, notamment dans l'épisode de la mort de Didon, contribuèrent à ancrer durablement le souvenir de ce personnage dans l'esprit de saint Augustin. Elle relève l'association étroite qui existe entre mémoire et émotion, à travers l'empathie qui constitue un levier d'apprentissage d'une grande efficacité. Ce moyen mnémotechnique de premier choix permet à la fois que les jeunes élèves acquièrent la connaissance de ce texte classique mais également qu'ils apprennent la langue latine, principale préoccupation des pédagogues médiévaux. Les gloses qui ponctuent abondamment le texte de l'Énéide, dans les manuscrits étudiés par M. Curry Woods, se font denses dans les moments où le discours de Didon est chargé sur le plan du pathos. Les passages où la reine s'exprime apparaissent particulièrement annotés et commentés en regard de ceux traduisant le discours d'Énée. Cette observation révèle la mise en valeur des émotions féminines dans le processus d'apprentissage inspiré par les manuscrits des textes classiques. Une notation musicale (les neumes) renforce de manière exacerbée les passages d'une grande intensité émotionnelle dans un ensemble de manuscrits copiés entre 900 et 1200 , se concentrant sur les voix féminines. Si les neumes ne sont plus utilisés dans les manuscrits postérieurs à 1200 pris en compte par M. C. Woods, cette emphase se prolonge sous une autre forme, à travers les notations des gloses. Par ce biais, les garçons sont autorisés à explorer et à vivre une série d'émotions et d'actions qui demeureraient du domaine de l'interdit ou de l'impossible en dehors de l'espace fictif des œuvres étudiées et de la salle de classe qui échappe au réel. L'expérience pour les jeunes hommes de l'identification au genre opposé s'inscrit dans la lignée de cette exploration transgressive.

Le second chapitre se concentre sur l'identité masculine telle qu'elle est construite dans les textes classiques, à travers des exemples de personnages masculins transmis aux écoliers médiévaux. Il est tout d'abord question du travestissement d'Achille dans l'Achilléide. L'épisode où Achille se déguise en femme pour échapper à la guerre est en effet enseigné dans les classes, notamment lorsqu'au XII ${ }^{\mathrm{e}} \mathrm{s}$. l'Achilléide commence à être largement employé comme texte scolaire. Les commentaires des manuscrits de l'Achilléide mettent en lumière la masculinité 
en tant qu'apprentissage et contribuent sans doute à enseigner le juste comportement sexué aux hommes en devenir. Si Achille est dans un premier temps élevé par un centaure, son déguisement en femme sous l'impulsion de sa mère est présenté comme une déviation de son chemin vers la masculinité selon M. C. Woods. Cet épisode reproduit à certains égards la désorientation momentanée quant à l'identité sexuée propre à l'âge des écoliers qui étudient ces textes. Il permet d'arrimer le processus d'identification des jeunes lecteurs tout en les rassurant sur leur masculinité dans cet état transitoire de l'adolescence. La situation d'incertitude de genre se trouve par la suite résolue par les deux mentors d'Achille, Ulysse et Diomède, qui vont faire de lui un homme.

Un autre texte souvent utilisé dans les classes médiévales, l'Iliade latine, vient contrebalancer cet épisode en offrant des modèles de personnages très masculins et très féminins, sans ambiguïté, selon les critères mis en place par les textes classiques. Si l'identité et les actions des personnages se trouvent distinguées de manière évidente entre les sexes, l'attention se concentre presque exclusivement sur les protagonistes masculins, sur les nombreuses batailles menées par des hommes et sur leur mort. Ces caractéristiques semblent confirmer l'orientation du texte vers des lecteurs masculins et offrir un espace propice à l'enseignement du vocabulaire épique. La diffusion des deux œuvres étudiées dans ce second chapitre, dont témoignent les nombreuses copies conservées en Europe, met en lumière la large circulation de ces modèles de comportement auprès des écoliers des derniers siècles du Moyen Âge.

La dernière partie de cette étude se penche sur l'expressivité que révèlent les manuscrits des textes classiques destinés aux écoles médiévales. Les instructions que recèlent les gloses sont autant d'indices en faveur d'une récitation à voix haute de ces textes selon une intonation et une gestuelle soigneusement établies. Les émotions contenues dans ces œuvres, soulignées par les commentaires médiévaux, étaient destinées à être gardées en mémoire tant par l'esprit que par le corps à travers une lecture collective. Si les écoliers ne jouaient probablement pas de véritables drames, ils incarnaient et intériorisaient cependant dans les classes ces textes et le discours des personnages. À cet égard, les gloses des manuscrits donnent une voie d'accès privilégiée pour saisir la manière dont les textes classiques étaient non seulement interprétés, mais aussi joués (performed) dans les lieux d'apprentissage. L'adoption de la juste tonalité et des gestes conseillés participe du processus d'identification des écoliers aux protagonistes et contribue à faire des textes classiques, à travers les émotions des personnages féminins, de «puissants outils pédagogiques » («potent pedagogical tools $»$ p. 144).

De nombreux exemples, qu'il s'agisse des longues reproductions des gloses avec le texte original ou des images de manuscrits commentés ponctuant cet ouvrage, viennent étayer de manière solide le propos de Weeping for Dido. Les illustrations aident à entrer dans cet univers intellectuel et pédagogique, en donnant à voir l'élaboration graphique de la pensée explorée. Si l'analyse de M. C. Woods est efficace et convaincante, une conclusion à la fin de chaque chapitre aurait permis de clarifier davantage les idées de 1'a. Ceci offrirait au lecteur une synthèse plus substantielle des nombreux exemples proposés au fil des pages, parmi lesquels il est parfois difficile de saisir la thèse défendue, en particulier dans le second chapitre. En outre, alors que les manuscrits étudiés datent d'une période comprise entre le XII ${ }^{\mathrm{e}}$ et le $\mathrm{XV}^{\mathrm{e}} \mathrm{s}$., aucune réflexion chronologique n'est esquissée quant à ce vaste laps de temps, proposant une évolution temporelle, une inflexion au fil des siècles ou constatant la continuité qui s'établit entre ces textes de manière explicite. Riche dans ses changements culturels, la période que reflètent les manuscrits étudiés, qui pourtant s'étale sur plusieurs siècles, semble former un tout monolithique au sein de cette étude, qui distingue bien davantage les textes que les périodes dans lesquelles ils s'inscrivent. La perspective que propose Weeping for Dido n'en demeure pas moins originale et probante dans la place qu'elle accorde aux émotions et aux identités de genre dans le processus pédagogique, renouvelant l'appréhension de la réception des textes classiques au Moyen Âge.

Anne-Lydie Dubois Université de Genève 